\title{
Retrieving cloud top height in the JEM-EUSO cosmic-ray observation system
}

\author{
Susana Briz ${ }^{1, a}$, Anna Anzalone ${ }^{2,5}$, Francesco Isgrò ${ }^{3,5}$, Roberto Cremonini ${ }^{4}$, Ilaria Tabone ${ }^{4}$, Mario Bertainav ${ }^{4}$, \\ Irene Rodríguez ${ }^{1}$, Isabel Fernández-Gómez ${ }^{1}$, and Antonio J. de Castro ${ }^{1}$ for JEM-EUSO Collaboration \\ ${ }^{1}$ Universidad Carlos III de Madrid, Avda. de la Universidad 30, 28911 Leganés, Spain \\ ${ }^{2}$ INAF-IASF, Istituto di Astrofisica Spaziale e Fisica Cosmica, Palermo, Italy \\ ${ }^{3}$ DIETI, Università degli Studi di Napoli Federico II, Italy \\ ${ }^{4}$ Dipartimento di Fisica, Università degli Studi di Torino \& INFN Torino, Italy \\ ${ }^{5}$ Istituto Nazionale di Fisica Nucleare - Sezione di Catania and Napoli, Italy
}

\begin{abstract}
The main telescope of JEM-EUSO will determine Ultra High Energy Cosmic Ray properties by measuring the UV fluorescence light generated in the interaction between the cosmic rays and the atmosphere. Therefore, cloud information is crucial for a proper interpretation of the data. JEM-EUSO will observe the clouds in the field of view of the telescope making use of an atmospheric monitoring system, which consists of a LIDAR and an IR Camera. To retrieve the cloud top height from IR images two different methodologies will be used. The first one is based on stereo vision algorithms and requires two different views of the same scene. The second one is based on the relationship between the cloud top temperature and the cloud top height. From the IR camera the brightness temperature can be retrieved, thus the developed Split Window Algorithm retrieves the real cloud temperature from the brightness temperature in two spectral bands. This article presents a preliminary work, in which both methodologies are compared with the height given by MODIS. The stereo system is provided by the two geostationary satellites MSG-2 and MSG-3. The Split Window Algorithm has been tested in MODIS images of bands 31 and 32. This initial work is a first step to compare a very simplified version of both methodologies.
\end{abstract}

\section{Introduction}

JEM-EUSO [1] is the first space mission devoted to the exploration of the Universe through the detection of Extreme Energy (E $>60 \mathrm{EeV}$ ) Cosmic Rays (EECRs). Cosmic rays are observed by looking downward from the International Space Station (ISS) at the altitude of about $400 \mathrm{~km}$ from ground. JEM-EUSO will observe UV photon tracks produced by EECRs developing in the atmosphere and producing Extensive Air Showers (EAS).

Clouds affect the Cherenkov signal received from EAS, as well as its reconstruction efficiency. Estimation accuracy depends on cloud coverage and cloud top height $(\mathrm{CTH})$. The strength of the fluorescent light also depends on the transparency of the atmosphere, on the cloud coverage and on the height of the cloud top [2].

There are many satellites that provide atmospheric information from multi-spectral observations with good spatial and temporal resolutions, for instance, geostationary satellites (GOES, MSG, etc.), LEO satellites (Terra/Aqua, HIRS, etc.), CALIPSO, etc. However JEMEUSO requires spatially and temporally simultaneous information of those atmospheric parameters.

In order to monitor the atmospheric conditions and the cloud coverage in the JEM-EUSO FoV a state-of-art Atmospheric Monitoring System (AMS) will be included in the system [3]. The AMS is crucial to estimate the effective exposure with high accuracy and to increase the confidence level in the EECR events.

\footnotetext{
a e-mail: sbriz@fis.uc3m.es
}

The AMS consists of a LIDAR, an infrared (IR) camera, the slow-data of the main telescope and Numerical Weather Prediction models (NWP) to measure the CTH with an accuracy of about $500 \mathrm{~m}$. The LIDAR will measure the optical depth profiles of the atmosphere in selected directions. The IR camera will provide the cloud coverage and the cloud top temperature. The slow-data will give a picture in UV of the observed scene. The NWP models will be used to retrieve the atmospheric parameters (temperature, pressure and humidity vertical profiles) in the monitored region.

This work focuses on the algorithms to retrieve the $\mathrm{CTH}$ from the data measured by the IR camera. Cloud top height retrieval can be fulfilled using either accurate radiometric information or stereo vision algorithms. The first methodology is based on the relationship between the cloud top temperature and the cloud top height and uses one or two different spectral bands in the infrared window; the second one requires two different views of the same scene with the same IR band. The satellites that are able to fulfil these requirements will be described in the introduction of Sect. 2, while the two methodologies will be explained in detail respectively in Sects. 2.1 and 2.2. Comparisons and the achieved results on their simplified version are described in Sect. 3.

\section{Data and methods}

The algorithms that are developed for JEM-EUSO observing system have been applied to data from available 


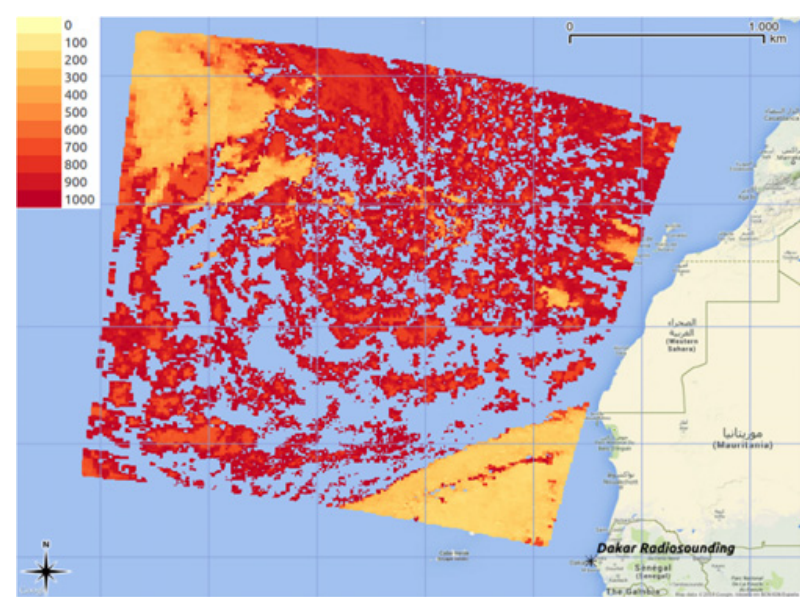

Figure 1. Cloud Top Pressure (hPa) provided by MODIS MOD_06 Level 2 Cloud Product.

operational meteorological satellites and compared with state-of-the-art CTH retrieval algorithms [4]. To apply the stereo vision technique, simultaneous observations from the geostationary Meteosat Second Generation (MSG) satellites MSG-3 and MSG-2 have been analysed. In fact these satellites are located on the Equatorial plane respectively at $0^{\circ}$ and $9.5^{\circ} \mathrm{E}$ at about $36,000 \mathrm{~km}$ altitude. MSG-3 fits out an entire scan of Europe and Africa every 15 minutes, while the Rapid Scan System of MSG-2 provides data for the Northern Hemisphere every 5 minutes, providing two different sights of view for the same area. To apply the radiative method, the Moderate Resolution Image Spectroradiometer (MODIS) has also been used; its IR resolution is about $1 \mathrm{~km}$ for MOD_02 Level-1B Calibrated Geolocated Radiances and $5 \mathrm{~km}$ for MOD_06 Cloud Products. This radiometer is integrated on Terra satellite and it provides data in 36 spectral bands, two of which (channel 31-32) have been used by the SWA radiative algorithm.

For comparison purposes a cloudy scene observed by MODIS, MSG-2 and MSG-3 has been selected. The difference in time between MODIS and MSG scans are respectively 6 and 8 minutes. The considered area is located at $19 \mathrm{~N}-38 \mathrm{~N}$ latitude and $15 \mathrm{~W}-36 \mathrm{~W}$ longitude (near Atlantic African coasts). Figure 1 shows cloud top pressure (CTP) [4], retrieved from MODIS on $2^{\text {nd }}$ April 2014 at 12:35 UTC. Low clouds in the centre and high clouds in the N-W and S-E corners can be easily noticed. Clouds cover about $48 \%$ of the image. The $75 \%$ of the cloudy area correspond to water clouds and $60 \%$ of ice clouds, which are considered thick with an optical depth $>2$. About $13 \%$ of the scene is filled by unknown-phase clouds.

\subsection{Radiative methods}

The approach to retrieve the cloud top height (CTH) based on radiative methods uses the relation between the temperature and the height along the vertical profile of the atmosphere to retrieve the cloud height from the cloud temperature. However the IR camera does not measure the cloud temperature directly. Instead, the IR camera measures the IR radiation coming from all the elements in its field of view (Earth surface, atmosphere and clouds). After calibration the radiance can be converted into temperature, which is called brightness temperature. Nevertheless, due to atmospheric effects (gases absorption/emission) the IR radiance emitted by the clouds is not the one received by the IR camera. Therefore the brightness temperature retrieved from measured radiance is not the temperature of the cloud. To correct the atmospheric effects and obtain the cloud temperature some algorithms can be applied. In a preliminary work several algorithms were designed and compared. Some of them were based in a monoband option and others are based on two spectral bands. Given the better results of the bi-spectral option (almost one order of magnitude), the algorithm applied in this work is a splitwindow algorithm (SWA). It uses two spectral bands in the 10-12 $\mu \mathrm{m}$ spectral region [5] and [6]. This algorithm was designed to retrieve the CTT from IR camera images. Therefore, the width and centre of the algorithm spectral bands are those of the JEM-EUSO IR camera (two $1 \mu \mathrm{m}$ width spectral bands centred in 10.8 and $12 \mu \mathrm{m}$ ). Since MODIS provides the brightness temperatures in these same spectral bands ( 31 and 32 channels), the algorithm can also be applied to MODIS images to retrieve the CTT. Then the CTT retrieved by the algorithm and the CTT delivered by MODIS as a product can be compared. This comparison will provide an indicator of the goodness of the CTT retrieved by the SWA. Equation (1) shows this algorithm,

$$
T_{\text {cloud }}\left(\mathrm{B}_{1} \mathrm{~B}_{2}\right)=-0.53819+2.6331 T_{B 1}-1.6305 T_{B 2}
$$

where $T_{\text {cloud }}$ is the cloud top temperature retrieved by the SWA, $T_{B 1}$ is the brightness temperature in the band centred in $10.8 \mu \mathrm{m}, T_{B 2}$ is the brightness temperature in the band centred on $12 \mu \mathrm{m}$.

The SWA is based on radiometric simulations of the physical problem [7]. The simulations were performed by means of the radiative transfer equation and MODTRAN atmospheric simulation code. Only scenarios where the cloud emissivity is 1 (thick clouds) have been considered which is a common situation and simplifies the radiative transfer equation. The same procedure has been used to simulate other IR scenarios to validate the algorithms. The difference between the cloud temperature used in these simulations and the temperature retrieved by the algorithm when applied to those scenarios is considered the algorithm error. The maximum retrieval error is associated to low clouds and atmospheres with high content of water vapour. But even for these cases the maximum error is $0.3 \mathrm{~K}$.

\subsection{Stereo vision method}

Satellite-based stereoscopy methods for CTH retrieval are independent from information regarding cloud/atmosphere parameters apart from the ones retrieved by analysing the images and the geometry of the stereo system. They are generally based on a multi view system with one or more cameras.

In the JEM-EUSO case the IR-camera besides measuring the IR radiation of a scene, works also as 


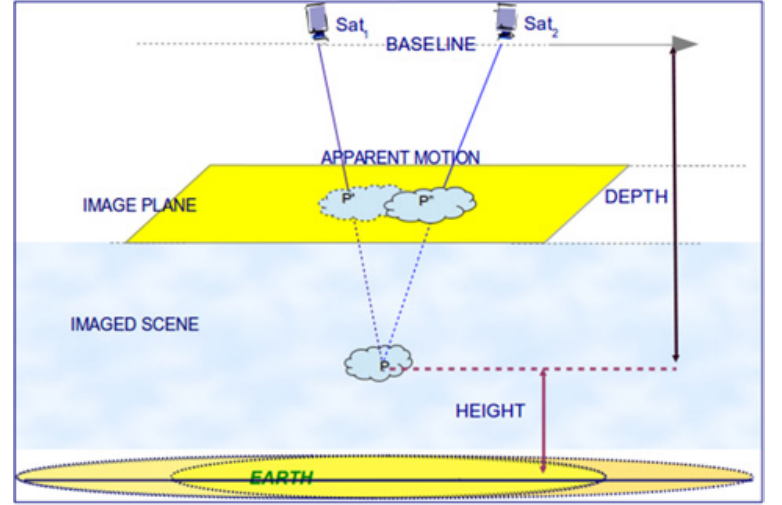

Figure 2. Stereo height estimation of $\mathrm{P} . \mathrm{P}^{\prime}$ and $\mathrm{P}^{\prime \prime}$ are associated by the matching step and intersecting the reconstructed optical rays, the height of $\mathrm{P}$ is retrieved. The overlap of the two images highlights the apparent movement of the cloud in the direction of the ISS motion.

a "stereo system" by exploiting the ISS movement. It acquires two views of the same sky area from different viewpoints and at different time.

In this paper a geostationary stereo system, has been considered to test a modified version of the algorithm [8], intended as one of the methods that will be developed to find the more suitable ones for JEM-EUSO.

The system is composed by the two MSG satellites mentioned in paragraph 2. Although the two imaging devices are synchronous, the spatial distance between the satellites is not enough to provide an accurate reconstruction of the height. Therefore it is expected that the whole range of heights of clouds present in the scene, will not be well reconstructed.

The main task of stereo retrieval is the matching step that identifies the same features in the two views and for each pixel the apparent motion (disparity) is estimated. The closer in time are the acquisition, the more accurate can be the matching. The final reconstruction is then accomplished by triangulating the reconstructed optical rays (Fig. 2).

\section{Results}

As a first step both methodologies, stereo vision and the SWA, have been applied to available and appropriate sensors to check their performance.

\subsection{SWA results}

In order to check the performance of the SWA to retrieve CTT in real scenarios (not only simulations), the algorithm has been applied to MODIS images. From all the products that MODIS provides we have used only some of them: brightness temperatures in channels 31 and 32 (IR camera bands), the cloud temperature, the emissivity and the cloud phase.

The SWA has been applied to a MODIS image taken from [4] at 12:35 located at the coordinates aforementioned. To retrieve the CTH from CTT close

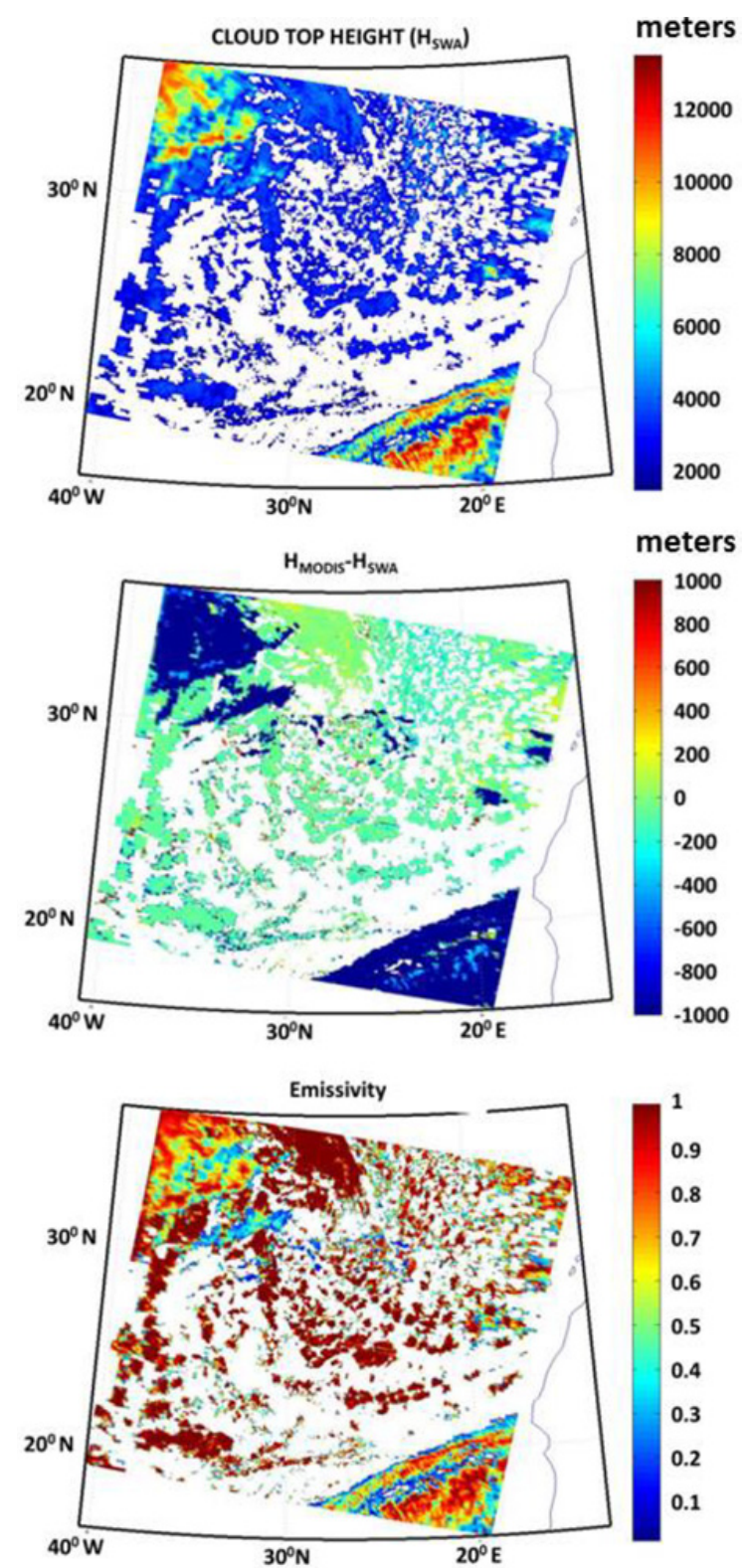

Figure 3. Height calculated by the procedure based on the SWA (up), difference between the heights obtained with the SWA and MODIS products (centre) and emissivity provided by MODIS (down).

in time vertical atmospheric profiles of Dakar have been used (61641 GOOY Dakar Observations at 12Z 02 Apr. 2014). The height retrieved by this procedure and the comparison between the height obtained directly from MODIS and from the temperature retrieved by the SWA is shown in Fig. 3 (up and centre respectively). From these two images it is observed that the height difference is small (lower than $200 \mathrm{~m}$ for clouds lower than $5000 \mathrm{~m}$ ). However, the height difference for higher clouds increases notably until $1000 \mathrm{~m}$. In order to explain this behaviour the cloud phase and emissivity products by MODIS have been considered. The image displayed in Fig. 3 (down) shows the emissivity provided by MODIS. Pixels of low emissivity show the highest errors. Since these low emissivity pixels are associated to ice phase it is clear that 


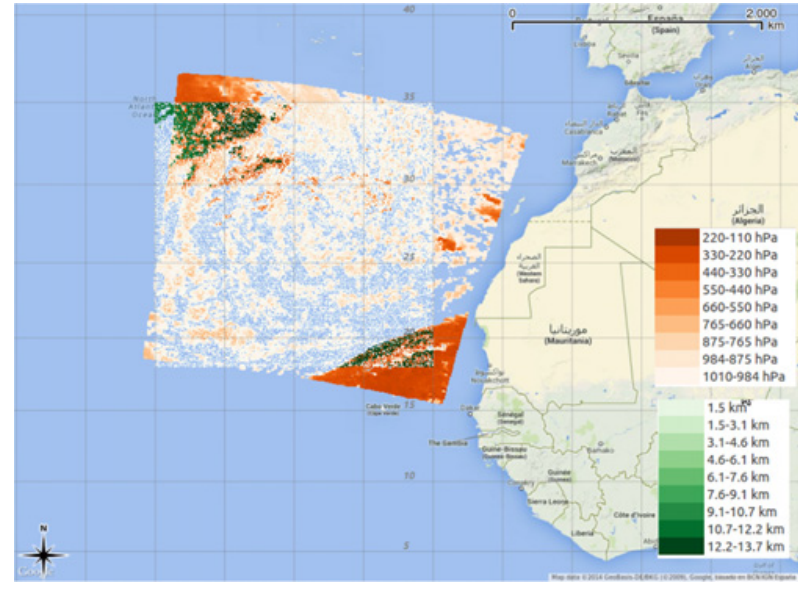

Figure 4. MSG stereo heights overlapped to MODIS heights.

the SWA is able to retrieve the temperature of water thick clouds with good accuracy although it is not appropriate to retrieve the temperature of ice thin clouds. This behaviour was expected since the algorithms were defined under the hypothesis of emissivity 1 .

\subsection{Stereo results}

The results of the stereo retrieval for the MSG-2 and MSG-3 stereo pair show that, as expected, the algorithm in this case fails to retrieve the height for the lowest clouds, because of geometric limits of the stereo system considered (too short baseline). With respect to the estimate of the CTH given by MODIS the results seem to overestimate the values, however it must be considered the data acquisition between the two systems is not synchronous, and that MODIS does not a provide the exact true height, although it is used for comparison due to its high performance.

\section{Conclusions}

The SWA is able to retrieve the temperature of thick water clouds with good accuracy, much lower than the $500 \mathrm{~m}$ required by JEM-EUSO mission, but it is not applicable to thin clouds, pixels at the cloud edge (low effective emissivity) or ice thin clouds, as expected since the SWA was designed under the hypothesis of emissivity 1 (thick water clouds). For pixels of low emissivity another algorithm is being designed. This alternative algorithm retrieves first the emissivity and then the cloud temperature and it is under validation process.
In this MSG stereo configuration, the stereo algorithm is able to retrieve the height of the highest clouds, with values highest to the ones of MODIS. The lowest clouds do not have detectable disparities and cannot be distinguished from ground, therefore are not assigned an altitude. However this must not be considered a strong limit for the problem here (JEM-EUSO) as the final system will have a proper baseline and the algorithm will be applied to other stereo sets of data to be evaluated.

This is the first time that two techniques that can be applied to JEM-EUSO IR camera data to retrieve the $\mathrm{CTH}$, are applied to the same scenario for comparison purposes. Although preliminary, the results show that both techniques are useful and complementary. Future working lines are: using another sensor with better baseline for stereo vision, including retrieval techniques to estimate thin cloud emissivity and temperature and considering a Mesoscale Atmospheric Model (Weather Researach and Forecasting, WRF Model) to retrieve the CTH from CTT instead of radiosoundings.

The Spanish Consortium involved in the JEM-EUSO Space Mission is funded by MICINN under projects AYA200906037-E/ESP, AYA-ESP 2010- 19082, AYA2011-29489-C03-01, AYA2011-29489-C03-03, AYA2012-39115-C03-01, AYAESP 2011-29489-C03, AYA-ESP2012-39115-C03-02, CSD200900064 (Consolider MULTIDARK) and by Comunidad de Madrid (CAM) under project S2009/ESP-1496. This work has been partially supported by the Italian Ministry of Foreign Affairs, General Direction for the Cultural Promotion and Cooperation.

\section{References}

[1] T. Ebisuzaki et al., Adv. Space Res. 53, 1499-1505 (2014)

[2] G. Sáez-Cano et al., Adv. Space Res. 53, 1536-1543 (2014)

[3] M. Rodriguez-Frias, EPJ Web of Conf. 53, 10005-p.17 (2013)

[4] W. Menzel, R. Frey, B. Baum, Cloud Top Properties and Cloud Phase - ATBD, MODIS Theor. Basis (2013)

[5] T. Inoue, J. Geophys. Res. 92, 3991 (1987)

[6] T. Nauss, A.A. Kokhanovsky, T.Y. Nakajima, Reudenbac, C. and J. Bendix, Atmos. Res. 78, 46 (2005)

[7] S. Briz, A.J. de Castro, I. Fernández-Gómez, I.Rodríguez, F. López, J. App. Rem. Sens. 8, 084990p.1-15 (2014)

[8] K.F. Manizade, J.D. Spinhirne and R. S. Lancaster, IEEE Trans. Geosci. Remot. Sen., 44: 9, p. 2481-2491 (2006) 